\title{
Editorial: The Evolving Geomagnetic Field
}

\author{
Greig A. Paterson ${ }^{1 *}$, Christopher J. Davies ${ }^{2}$ and Ron Shaar ${ }^{3}$ \\ ${ }^{1}$ Department of Earth, Ocean and Ecological Sciences, University of Liverpool, Liverpool, United Kingdom, ${ }^{2}$ School of Earth \\ \& Environment, University of Leeds, Leeds, United Kingdom, ${ }^{3}$ The Institute of Earth Sciences, The Hebrew University of \\ Jerusalem, Jerusalem, Israel
}

Keywords: paleomagnetism, archeomagnetism, geodynamo modeling, geomagnetism, core dynamics, secular variation, global magnetic field models, time-average fields

\section{Editorial on the Research Topic}

\section{The Evolving Geomagnetic Field}

Earth's magnetic field, generated over $2,800 \mathrm{~km}$ below the surface in the liquid core, is a long-lived and complicated feature of our planet that requires a multi-stranded approach to quantify and comprehend. From its early beginning in the Archean to the modern day, the geomagnetic field exhibits striking spatial and temporal behavior across a vast range of scales, including sustained periods of atypical stability (superchrons), polarity reversals, rapid and spatially localized intensity "spikes," and impulsive changes in field strength and direction such as geomagnetic jerks.

Characterizing and understanding these complex variations requires a diverse approach that synthesizes experimental, observational, theoretical, and computational approaches. In this collection of papers that contribute to the topic "The Evolving Geomagnetic Field," we bring together studies on secular variation, geodynamo simulations, paleomagnetism, archeological magnetism, magnetic field modeling, and sedimentary magnetism to shed light on the latest advances in exploring our magnetic field.

Our collection opens with Muxworthy who explores the latitudinal dependence of the magnetic field strength (paleointensity) over the last 5 Myrs. Building on existing models (Tauxe and Kent, 2004), Muxworthy demonstrates that models where non-axial dipole terms time-average to zero are incapable of capturing the variability of the observed paleointensity data. He concludes that the current paleointensity data requires a constant axial quadrupole term of up to $-10 \%$ of the axial dipole combined with an octupole term of $-15 \%$ of the dipole. Interestingly, this octupole term has the opposite sign to the axial dipole, which contrasts with estimates from directional studies and suggests that time-average field models should incorporate full vector analysis. Driscoll and Wilson also consider long-timescale behavior by utilizing geodynamo simulations to explore time-averaging of the field to isolate a dominant axial dipole. Their analysis suggests that averaging over 20-120 kyr is required to obtain stable paleomagnetic poles, but for a frequently reversing field, longer periods may be needed. Obtaining reliable averages of the axial dipole moment, however, requires longer time periods and intensity estimates from less stable fields may introduce a latitudinal bias away from the true axial dipole moment, which may be corrected for if the reversal rate is known.

On shorter millennial timescales, archeomagnetic studies in China flourished in the 1980's and 90's, but went through a period of quiescence for more than a decade. Cai et al. review the rejuvenation of Chinese archeomagnetism over the past 5 years and present the state-of-the-art knowledge for how Earth's magnetic field has varied across China for the last $\sim 7000$ years. From the most extensive collection to-date, Cai et al. construct a new series of archeomagnetic master curves for China, which will serve as an invaluable dating tool for Chinese archeologists. 
On similar timescales, but the other side of the world, archeomagnetism in the Middle East continues to produce valuable results as exemplified by Shaar et al., who compile the first catalog of archeomagnetic directional data from Israel, consisting of 76 directions. When pooled together with directional data from nearby regions, strong deviations from an axial dipole field are observed at 800-1000 BCE, lending further support to anomalous field behavior at this time, known as the Levantine Iron Age Anomaly (LIAA). The LIAA is also the focus of Korte and Constable, who investigate the two rapid spikes in field intensity during this time period using a new series of global magnetic field models. By preferentially up-weighting or downweighting data to construct their models, they conclude that these rapid field changes are the result of the growth and decay of quasistationary flux patches superposed on a stronger, but variable dipole. Requiring axial dipole variations no more that $60 \%$ higher than the present day, their analyses suggest that the intensity changes associated with the LIAA may be less anomalous than previously thought.

Yamamoto and Yamaoka turned to lava flows from the island of Hawaii to obtain absolute paleointensity over the last $\sim 24 \mathrm{kyr}$, extending the time range of archeomagnetic studies. The results of Yamamoto and Yamaoka were obtained from the TsunakawaShaw paleointensity method, which is based on a specimen's coercivity spectrum. These new results are consistent with those from the IZZI method (Cromwell et al., 2015, 2018), which are based on a specimen's blocking temperature spectrum. This agreement between two distinct methods boosts our confidence in their overall reliability and is a great example of different approaches complimenting each other to yield robust records.

The final two contributions to this Research Topic use sedimentary sequences as detailed recorders of geomagnetic field

\section{REFERENCES}

Cromwell, G., Tauxe, L., Staudigel, H., and Ron, H. (2015). Paleointensity estimates from historic and modern Hawaiian lava flows using glassy basalt as a primary source material. Phys. Earth Planet. Interiors 241, 44-56. doi: 10.1016/j.pepi.2014. 12.007

Cromwell, G., Trusdell, F., Tauxe, L., Staudigel, H., and Ron, H. (2018). Holocene paleointensity of the Island of Hawai ' $\mathrm{i}$ from glassy volcanics. Geochem. Geophys. Geosyst. 19, 3224-3245. doi: 10.1002/2017GC 006927

Tauxe, L., and Kent, D. V. (2004). "A simplified statistical model for the geomagnetic field and the detection of shallow bias in paleomagnetic inclinations: Was the ancient magnetic field dipolar?," in Timescales of the behavior. Firstly, Lund brings together marine and lake sediment records from six regions of the world, spanning the Americas and the Philippines/Indonesia and dating back to $\sim 70 \mathrm{ka}$. Lund not only shows that the records are regionally consistent, but that the variability of the records is generally globally coherent with relative paleointensity having the highest consistency. Finally, Nilsson et al. focus on varved lake sediments from Sweden to develop and test a new Bayesian method to account for sedimentary lock-in depth (the depth to which sediments must be buried to "freeze-in" their magnetic recording). This is a potentially valuable new tool, not only to understand the lockin process of sedimentary records, but also for incorporating sedimentary records in long-term models of field behavior, such as those of Korte and Constable, which are part of the family of related models that have been used here by Nilsson et al. themselves, but also by Cai et al., Shaar et al., and Yamamoto and Yamaoka.

The breadth and inter-connectivity of the science presented here is the tip of the large iceberg that is the study of the geomagnetic field. We hope that the articles compiled in this Research Topic provide an enjoyable read that stimulates valuable discussion. These contributions have pushed forward our understanding of field behavior across a range of timescales, but also new questions and challenges. All of which are the seeds for new collaborations and renewed understanding of our magnetic field.

\section{AUTHOR CONTRIBUTIONS}

All authors listed have made a substantial, direct and intellectual contribution to the work, and approved it for publication.

Paleomagnetic Field, eds J. E. T.Channell, D. V. Kent, W. Lowrie, and J. G. Meert (Washington, DC: AGU), 101-115.

Conflict of Interest Statement: The authors declare that the research was conducted in the absence of any commercial or financial relationships that could be construed as a potential conflict of interest.

Copyright (c) 2019 Paterson, Davies and Shaar. This is an open-access article distributed under the terms of the Creative Commons Attribution License (CC BY). The use, distribution or reproduction in other forums is permitted, provided the original author(s) and the copyright owner(s) are credited and that the original publication in this journal is cited, in accordance with accepted academic practice. No use, distribution or reproduction is permitted which does not comply with these terms. 